\title{
Temperature and entropy of Schwarzschild-de Sitter space-time
}

\author{
S. Shankaranarayanan* \\ DCTD, University of Azores, 9500 Ponta Delgada, Portugal.
}

\begin{abstract}
In the light of recent interest in quantum gravity in de Sitter space, we investigate semi-classical aspects of 4-dimensional Schwarzschild-de Sitter space-time using the method of complex paths. The standard semi-classical techniques (such as Bogoliubov coefficients and Euclidean field theory) have been useful to study quantum effects in space-times with single horizons; however, none of these approaches seem to work for Schwarzschild-de Sitter or, in general, for space-times with multiple horizons. We extend the method of complex paths to space-times with multiple horizons and obtain the spectrum of particles produced in these space-times. We show that the temperature of radiation in these space-times is proportional to the effective surface gravity - inverse harmonic sum of surface gravity of each horizon. For the Schwarzschild-de Sitter, we apply the method of complex paths to three different coordinate systems - spherically symmetric, Painleve and Lemaitre. We show that the equilibrium temperature in Schwarzschild-de Sitter is the harmonic mean of cosmological and event horizon temperatures. We obtain Bogoliubov coefficients for space-times with multiple horizons by analyzing the mode functions of the quantum fields near the horizons. We propose a new definition of entropy for space-times with multiple horizons analogous to the entropic definition for space-times with a single horizon. We define entropy for these space-times to be inversely proportional to the square of the effective surface gravity. We show that this definition of entropy for Schwarzschild-de Sitter satisfies the D-bound conjecture.
\end{abstract}

PACS numbers: 04.70.Dy, 04.62.+v

\section{INTRODUCTION}

Over the last three decades, quantum field theory in de Sitter (dS) space has been a subject of growing interest. In the 1970's, the attention was due to the large symmetry group of dS space, which made the field theory in dS space less ambiguous than, for example, Schwarzschild space-time. In the 1980's, the focus was due to the role it played during inflation - accelerated expansion in the universe's distant past. Recent attention to dS and asymptotic dS space-times is motivated by two aspects: (i) Observations [1] suggest that the universe might be currently asymptotic $\mathrm{dS}$ and approach a pure dS space (ii) Success of AdS/CFT correspondence [2] has lead to the intense study of quantum gravity of de Sitter space [3]. The focus has been to obtain an analogue of the AdS/CFT correspondence in the dS space [3-5]. For recent attempts on the semi-classical aspects of $\mathrm{dS}$ and asymptotic dS space in the light of the dS/CFT correspondence, please see Refs. [6-10]. [The authors in Refs. $[6,7]$ extended the method introduced in Ref. [11] to the Painlevé coordinates of (Schwarzschild)dS.]

Even though, there has been an extensive study of the semi-classical aspects of dS (for a recent review, see Ref. [12]) very little has been understood in the case of Schwarzschild-de Sitter (SdS) space-time. (An incomplete list of references, as regard to semi-classical aspect, is given in Refs. [13-16].) The fundamental difference between SdS and dS (also Schwarzschild) space-times is the existence of multiple horizons. SdS has two - cosmolog-

${ }^{*}$ E-mail: shanki@notes.uac.pt ical and event - horizons, while dS (and Schwarzschild) space-time has only one horizon.

Various semi-classical approaches or techniques (such as Bogoliubov coefficient, particle detectors, effective action, Euclidean field theory) have been used in the literature to study quantum effects in space-times with single horizon (like dS and Schwarzschild space-times). All the approaches conclude that the notion of temperature (and entropy) of the space-time is associated with the horizon. In the case of Bogoliubov coefficients, one uses the mode functions to obtain the spectrum of particles while using Euclidean field theory, one obtains temperature using the periodicity arguments. Even though, these approaches work well for space-times with single horizon, none of them work for SdS or, in general, space-times with multiple horizons. A naive extension of these approaches to SdS leads us to the conclusions that the SdS has two different temperatures associated with the two horizons. Using this extension, it has been argued $[6,17]$ that the SdS will inevitably evolve towards an empty de Sitter space indicating that SdS may never be in thermodynamic equilibrium with a single temperature associated with the space-time.

The above argument seems to be in contradiction with the well known case - Schwarzschild black-hole in thermal equilibrium with a radiation in a bounded box. In this case, black-hole has a negative specific heat while the radiation has a positive specific heat. The two will be in thermal equilibrium if the box is bounded, in other words compact. On the contrary, if the box is unbounded the black-hole evaporates completely. The situation is identical to the case of our interest - black-holes in de Sitter space. The de Sitter space is compact with no notion of spatial infinity. Besides, it has a positive specific heat 
similar to the above mentioned case. The specific heat of de Sitter space is given by $[12,14]$

$$
C_{V}=\frac{\partial E}{\partial T}=\frac{1}{4 \pi T_{d S}^{2}}=\pi l^{2}=S_{d S}
$$

where $S_{d S}\left(T_{d S}\right)$ is the entropy (temperature) of de Sitter space. However, the difference between de Sitter and the bounding box is that the de Sitter has a (cosmological) horizon while the bounding box, by construction, does not posses a horizon. The similarity of the two systems strongly suggests that we should be able to obtain a temperature for SdS corresponding to system in thermal equilibrium.

Given this, one would like to ask the following question: Can one obtain a temperature for SdS which corresponds to system in thermal equilibrium using semiclassical techniques? The purpose of this paper is an attempt in this direction. As mentioned in earlier paragraphs, standard quantum field theoretic techniques have not proven useful for space-times with multiple horizons. In this paper, we extend the method of complex paths to space-times with multiple horizons and obtain the spectrum of particles produced in these space-times. (The method of complex paths has proved to be useful in obtaining the temperature associated with a quantum field propagating in a spherically symmetric coordinate spacetimes with single horizon [18-21].) We show that the temperature of radiation in these space-times is proportional to the effective surface gravity - inverse harmonic sum of surface gravity of each horizon. In the case of Schwarzschild-de Sitter, we apply the method of complex paths to three different coordinate systems - spherically symmetric, Painlevé and Lemaitre. We show that the equilibrium temperature in Schwarzschild-de Sitter is the harmonic mean of cosmological and event horizon temperature. We obtain Bogoliubov coefficients for spacetimes with multiple horizons by analyzing the mode functions of the quantum fields near the horizons.

We propose a new definition of entropy for space-times with multiple horizons analogous to the entropic definition for space-times with single horizon. We define the entropy for these space-times to be inversely proportional to the square of effective surface gravity. We show that this definition of entropy for SdS satisfies the D-bound conjecture [22].

The paper is organized as follows: In section II A, we discuss the general properties of spherically symmetric space-times. A brief description of SdS geometry is given in section II B. In section III A, we apply the method of complex paths to a general spherically symmetric spacetimes and show that the equilibrium temperature in proportional to the inverse harmonic sum of the surface gravity of each horizon. In sections III B and III C, we apply the method of complex paths to three coordinate systems - spherically symmetric, Painlevé and Lemaitre - of SdS. In section $\mathrm{V}$, we propose a new definition of entropy for space-times with multiple horizons and discuss its implications for SdS. Finally, in section VI, we discuss the results.

Throughout this paper, the metric signature we shall adopt is $(-,+,+,+)$. We use Greek letters for $(3+1)$-D and lower case Latin letters for $(1+1)$-D. Quantum field is a massless, minimally coupled scalar field $(\Phi)$.

\section{SPHERICALLY SYMMETRIC SPACE-TIMES}

\section{A. General analysis}

The line-element for an interval in a spherically symmetric space-time can be written in the following forms:

$$
\begin{aligned}
d s^{2} \equiv & g_{\mu \nu}^{(4)} d x^{\mu} d x^{\nu} \\
= & g_{a b}^{(2)} d x^{a} d x^{b}+\exp \left[-2 \phi\left(x^{a}\right)\right] d \Omega^{2} \\
= & -\exp \left[\nu\left(x^{0}, x^{1}\right)\right]\left(d x^{0}\right)^{2}+\exp \left[\lambda\left(x^{0}, x^{1}\right)\right]\left(d x^{1}\right)^{2} \\
& +\exp \left[-2 \phi\left(x^{0}, x^{1}\right)\right] d \Omega^{2} .
\end{aligned}
$$

where $d \Omega^{2}$ is the $2-$ dimensional angular line element. As discussed in Ref. [23] (see also Refs. [19]), the spacetime structure of spherically symmetric space-times can be understood via $R$ and $T$ regions. If at the given event in the coordinate system (3), the inequality

$$
\exp [\nu-\lambda]>\left(\frac{\partial \phi}{\partial x^{0}} / \frac{\partial \phi}{\partial x^{1}}\right)^{2}
$$

is satisfied, then the event is defined as $R$-region. [If the above inequality is satisfied at a certain world point then by the virtue of the continuity $\left(\exp (\nu-\lambda), \partial \phi / \partial x^{0}\right.$, $\partial \phi / \partial x^{1}$ cannot be discontinuous) it is satisfied in some neighborhood of this point. Thus, the points in the neighborhood of this system of coordinates satisfies the above inequality are $R$-points and a set of them a $R$-region.] If the opposite inequality is satisfied, the event is in a $T$ region. The definitions of $R$ and $T$ regions can be shown to be coordinate invariant.

Spherically symmetric coordinate: Choosing the Schwarzschild gauge, line-element (3) can be written as

$$
d s^{2}=-g(r) d t^{2}+\frac{d r^{2}}{g(r)}+r^{2} d \Omega^{2},
$$

where $g(r)$ is an arbitrary (continuous, differentiable) function of $r$. For space-times with single horizon (like Schwarzschild, dS), $g(r)$ vanishes at one point say $r=r_{0}$. Near $r_{0}, g(r)$ can be expanded as

$$
g(r)=R\left(r_{0}\right)\left(r-r_{0}\right)
$$

where $R\left(r_{0}\right)$ is twice the surface gravity $(\kappa)$ of the horizon.

For space-times with multiple horizons (like SdS), $g(r)$ vanishes at more than one point say $r=r_{i}$ where $i=1,2, \cdots, n$. In general, $g(r)$ can be written in the following form

$$
g(r)=a \frac{\left(r-r_{1}\right)\left(r-r_{2}\right)\left(r-r_{3}\right) \cdots\left(r-r_{n}\right)}{r^{m}},
$$


where $a$ is a constant, $m<n, r_{n}>r_{n-1}>\cdots>r_{1}$ and all $r_{i}$ 's are assumed to be positive. Around each of these points one can expand $g(r)=R\left(r_{i}\right)\left(r-r_{i}\right)$ where $R\left(r_{i}\right) / 2$ is the surface gravity $\left(\kappa_{i}\right)$ of each of these horizons.

$R(T)$ region in the spherically symmetric coordinate system satisfy the inequality condition $g(r)>0(g(r)<$ $0)$. For space-times with multiple horizons, since $g(r)$ has multiple zeros, there are multiple $R$ and $T$ regions.

Painlevé coordinate: In order to obtain a line element which is regular at the horizon, we define a new time coordinate $\left(t_{P}\right)$ which is related to static time coordinate $(t)$ by the relation

$$
t=t_{P} \pm f(r),
$$

where $f$ is required to be a function of $r$ alone to ensure that the metric remains stationary $[6,7]$. The form of $f(r)$ can be obtained by imposing the condition that the resulting metric be regular at the horizon. This can be realized by demanding that the constant-time slices be flat, i. e.,

$$
\frac{1}{g(r)}-g(r)\left[\frac{d f}{d r}\right]^{2}=1 \Longrightarrow \frac{d f}{d r}=\frac{\sqrt{1-g(r)}}{g(r)}
$$

Substituting the expressions for $f(r)$ and $t$ in (5), we get

$$
d s^{2}=-g(r) d t_{P}^{2} \pm 2 \sqrt{1-g(r)} d r d t_{P}+d r^{2}+r^{2} d \Omega^{2} .
$$

We will refer to the above line-element as Painlevé coordinate. The above line element is a stationary - but not a static - system. "+" sign in the cross-term corresponds to the ingoing null geodesic while the "-" sign corresponds to an outgoing null geodesic.

For both, in-going and out-going null geodesics, the inequality condition for the $R$ region is given by $g(r)>0$ implying that the whole of space-time is doubly mapped w. r. t. spherically symmetric coordinate system [19].

Lemâitre coordinate: We can get rid of the cross term, in line-element (10), by performing a transformation of the radial coordinate $(r)$. This can be achieved by demanding that a set of curves $\left[t_{P}, r\left(t_{P}\right)\right]$ whose tangent vectors $(1, d r / d \tau)$ are orthogonal to the surface of constant Painlevé time. This gives

$$
R \mp t_{P}=\int \frac{d r}{\sqrt{1-g(r)}}
$$

Substituting for $r$ in (10), we get

$$
d s^{2}=-d t_{P}^{2}+[1-g(r)] d R^{2}+r^{2} d \Omega^{2} .
$$

We will refer to the above line-element as Lemâitre coordinate. The line-element is explicitly dependent in time and test particles at rest relative to the reference system are particles moving freely in the given field. The coordinate system can be modeled as that natural to a freely in-falling/out-falling observer whose velocity at radial infinity is zero. ["-" sign in equation (11) corresponds to the in-falling observer while the + sign in equation (11) corresponds an out-falling observer.]

In terms of spherically symmetric coordinate time $(t)$, we have

$$
R \mp t=\int \frac{d r}{g(r)} \frac{1}{\sqrt{1-g(r)}} .
$$

From equation (11) it is can be seen that

$$
r=\text { function of }\left(R \mp t_{P}\right) \text {. }
$$

For the upper sign in (11), defining $1-g(r)=F(U)$ and $r^{2}=G(U)$, the Lemaitre line element (12) in terms of the light cone coordinates $(U, V)\left[U \equiv R-t_{P}, V \equiv R-t_{P}\right]$ is

$$
\begin{aligned}
d s^{2} & =\left(\frac{F(U)-1}{4}\right)\left(d V^{2}+d U^{2}\right) \\
& +2\left(\frac{F(U)+1}{4}\right) d U d V+G(U) d \Omega^{2}
\end{aligned}
$$

For the line element corresponding to an in-falling observer, $R$ region is given by $F(U)>0$. For the $T$ region, the inequality is opposite. For an out-falling observer, the $R$ region is given by the inequality $F(V)>0$.

In the $T$ region there is an asymmetry in the direction of flow and hence the $T$ regions corresponding to the infalling and out-falling observer will be different while the $R$ regions will be the same. (For an elaborate discussion on this aspect, please refer to Refs. [19].) Hence, the $T$ region in the Lemaitre coordinates is a doubly mapped $T$ region of spherically symmetric coordinate [19].

\section{B. Schwarzschild-de Sitter}

The spherically symmetric coordinate of SdS spacetime is given by the line-element (5), where

$$
g(r)=\left(1-\frac{2 M}{r}-\frac{r^{2}}{l^{2}}\right)
$$

$M$ is the mass of the black-hole and $l^{2}$ is related to the positive cosmological constant. The space-time has more than one horizon if $0<y<1 / 27$ where $y=M^{2} / l^{2}$. The black hole horizon $\left(r_{h}\right)$ and the cosmological horizon $\left(r_{c}\right)$ are located respectively at

$$
\begin{aligned}
& r_{h}=\frac{2 M}{\sqrt{3 y}} \cos \frac{\pi+\psi}{3} \\
& r_{c}=\frac{2 M}{\sqrt{3 y}} \cos \frac{\pi-\psi}{3}
\end{aligned}
$$

where

$$
\psi=\cos ^{-1}(3 \sqrt{3 y})
$$

In the limit of $y \rightarrow 0$, we get $r_{h} \rightarrow 2 M$ and $r_{c} \rightarrow l$. [Note that $r_{h}<r_{c}$, i. e. event horizon is the smallest 


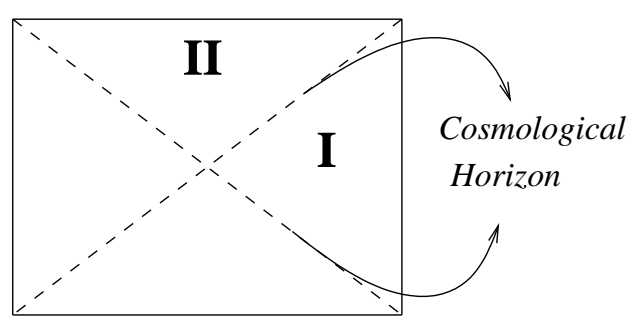

( A )
Curvature Singularity

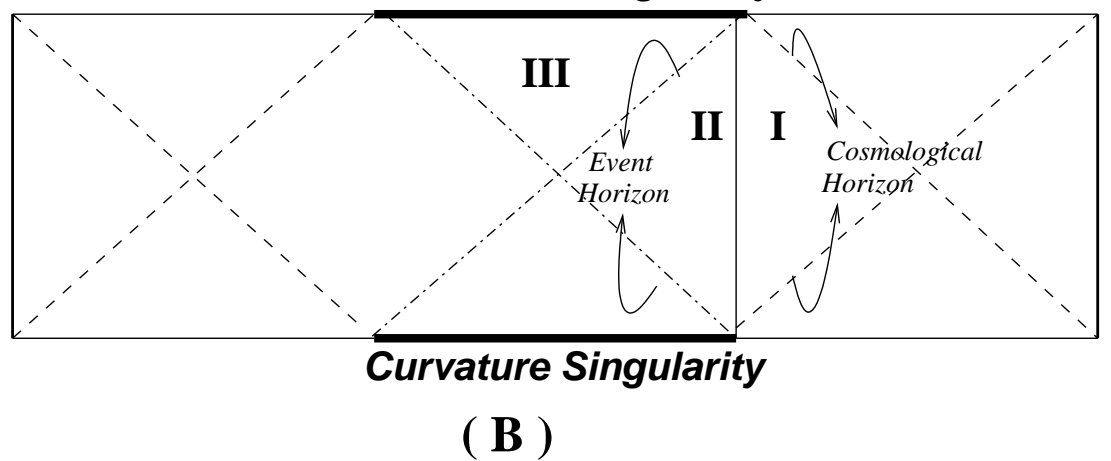

FIG. 1: Figure (A) is the Penrose diagram of the de Sitter space where the left and right sides are identified. Spherically symmetric coordinate system of de Sitter covers region (I) while the Painlevé and Lemaitre coordinate system cover regions (I) and (II). Figure (B) is the Penrose diagram illustrating the causal structure of the Schwarzschild de Sitter space-time. Here again, the left and right sides are identified. Spherically symmetric coordinate system covers regions (I) and (II). Painlevé coordinate system covers regions (II) and (III).

positive root.] The space-time is dynamic for $r<r_{h}$ and $r>r_{c}$. In the limit $y \rightarrow 1 / 27$, the two horizons - event and cosmological - coincide and is the well known Nariai space-time. If $y>1 / 27$, the space-time is dynamic for all $r>0$.

Surface gravity of these horizons are given by [24]

$$
\begin{aligned}
& \kappa_{h}=\alpha\left|\frac{M}{r_{h}^{2}}-\frac{r_{h}}{l^{2}}\right|, \\
& \kappa_{c}=\alpha\left|\frac{M}{r_{c}^{2}}-\frac{r_{c}}{l^{2}}\right|,
\end{aligned}
$$

where

$$
\alpha=\frac{1}{\sqrt{1-(27 y)^{1 / 3}}} .
$$

In the limit of $y \rightarrow 0$, we get $\kappa_{h} \rightarrow 1 /(4 M)$ and $\kappa_{c} \rightarrow 1 / l$. The range of $\kappa_{h}$ and $\kappa_{c}$ are

$$
\begin{gathered}
\frac{3 \sqrt{3}}{4 l}<\kappa_{h}<\frac{\sqrt{3}}{l} \Longrightarrow \frac{l}{\sqrt{3}}<\frac{1}{\kappa_{h}}<\frac{4 l}{3 \sqrt{3}}, \\
\frac{1}{l}<\kappa_{c}<\frac{\sqrt{3}}{l} \Longrightarrow \frac{l}{\sqrt{3}}<\frac{1}{\kappa_{c}}<l
\end{gathered}
$$

[The above ranges are obtained by setting $y \rightarrow 0$ and $y \rightarrow(1 / 27)$ in equations $(20,21)$.]

Figure (IB) illustrates one version of the Penrose diagram of SdS space-time. [For an easy comparison, we have also provided the Penrose diagram of de Sitter space.] The static coordinate system covers region (I) and (II) of the Penrose diagram. The boundaries of the static region consist of (i) past and future cosmological horizons, and (ii) past and future event horizons.
Painlevé line-element of SdS is given by

$$
d s^{2}=-g(r) d t_{P}^{2} \pm 2 \sqrt{\frac{2 M}{r}+\frac{r^{2}}{l^{2}}} d r d t_{P}+d r^{2}+r^{2} d \Omega^{2}
$$

where $t_{P}$ is related to $t$ by the relation

$$
t=t_{P} \pm \int d r \frac{\sqrt{2 M / r+r^{2} / l^{2}}}{\left(1-2 M / r-r^{2} / l^{2}\right.} .
$$

The above line element is a stationary - but not a static - system and it covers regions (II) and (III) of the Penrose diagram. "+" sign in the above chart is suitable for studying the physical experience of observers falling freely and radially into the hole. ["+" sign in the crossterm corresponds to the ingoing null geodesic while the "_" sign corresponds to an outgoing null geodesic.] For both, in-going and out-going null geodesics, the inequality condition for the $R$ region [23] is given by $g(r)>0$ implying that the whole of space-time is doubly mapped $w$. r. t. spherically symmetric coordinate system (5).

The Lemaitre line-element is given by

$$
d s^{2}=-d t_{p}^{2}+\left(\frac{2 M}{r}+\frac{r^{2}}{l^{2}}\right) d R^{2}+r^{2} d \Omega^{2}
$$

where,

$$
2 r^{3 / 2}=\left(2 M l^{2}\right) \exp \left[-\frac{3\left(R \pm t_{p}\right)}{2 l}\right]-\exp \left[\frac{3\left(R \pm t_{p}\right)}{2 l}\right] .
$$

The line-element is explicitly dependent in time and test particles at rest relative to the reference system are particles moving freely in the given field. $R-t_{P}$ in the R.H.S of the above equation corresponds to the in-falling 
observer while the $R+t_{P}$ in the R. H. S of the above expression corresponds to an out-falling observer. As like Painlevé, Lemaitre line-element also covers regions (II) and (III) of the Penrose diagram.

For the line element corresponding to an in-falling observer, $R$ region [23] is given by $F(U)>0\left(U=R-t_{P}\right)$. For the $T$ region, the inequality is opposite. For an outfalling observer, the $R$ region is given by the inequality $F(V)>0$.

\section{PARTICLE PRODUCTION IN SPACE-TIMES WITH MULTIPLE HORIZONS}

In Refs. [18-20] the method of complex paths was applied to space-times with single horizon. The temperature associated with the quantum field (in the three coordinate systems - spherically symmetric, Painlevé and Lemaitre) in these space-times were shown to be consistent with the temperatures obtained by using other quantum field theoretic techniques like Bogoliubov coefficients, Euclidean field theory, effective action. As discussed in Introduction, the standard quantum field theoretic techniques do not work well for space-times with multiple horizons. In this section, we extend the method of complex paths to space-times with multiple horizons and in particular to Schwarzschild-de Sitter space-time. We show that the effective temperature for the quantum fields in these space-times is proportional to the inverse harmonic sum of surface gravity at each horizon.

\section{A. General spherically symmetric space-time}

In this subsection, we obtain the effective temperature in a general spherically symmetric space-time with multiple horizons. [The result, in this subsection, is due to Ref. [26] and is summarized here for completeness.]

Consider a quantum field propagating in a general spherically symmetric space-time (5) with multiple horizons. The wave equation is given by

$$
\begin{aligned}
\partial_{\mu}\left(\sqrt{-g} g^{\mu \nu} \partial_{\nu}\right) \Phi & =0, \\
\frac{r^{2}}{g(r)} \frac{\partial^{2} \Psi}{\partial t^{2}}-\frac{\partial}{\partial r}\left(r^{2} g(r) \frac{\partial \Psi}{\partial r}\right)+L^{2} \Psi & =0 .
\end{aligned}
$$

where $\Phi\left(x^{\mu}\right)=\Psi(t, r) Y_{l m}(\theta, \phi)$. The semi-classical wave-functions satisfying the above are obtained by making the ansatz

$$
\Psi(r, t)=\exp \left[\frac{i}{\hbar} S(r, t)\right]
$$

where $S$ is a functional which will be expanded in powers of $\hbar$. Expanding $S$ in a power series of $\hbar$ :

$$
S(r, t)=S_{0}(r, t)+\hbar S_{1}(r, t)+\hbar^{2} S_{2}(r, t)+\ldots,
$$

and substituting the resulting expression in equation (29) (neglecting terms of order $\hbar$ or higher), we have

$$
-\frac{1}{g(r)}\left(\frac{\partial S_{0}}{\partial t}\right)^{2}+g(r)\left(\frac{\partial S_{0}}{\partial r}\right)^{2}+\frac{L^{2}}{r^{2}}=0 .
$$

This is simply the Hamilton-Jacobi equation satisfied by a massless particle moving in a general spherically symmetric coordinate. Making the ansatz $S_{0}=-E t+B(r)$, we get

$$
\begin{aligned}
B(r) & = \pm \int^{r} \frac{d r}{g(r)} \sqrt{E^{2}-\left(L^{2} / r^{2}\right) g(r)} \\
& = \pm \frac{E}{a} \int^{r} d r \frac{r^{m}}{\left(r-r_{1}\right)\left(r-r_{2}\right) \cdots\left(r-r_{n}\right)}
\end{aligned}
$$

[The last expression is obtained by setting $L=0$ by noticing that near the horizon, the presence of the $L^{2}$ term can be neglected. It can also be noted that the above expression is valid for an arbitrary dimension and hence the following result is true for a spherically symmetric space-time in an arbitrary dimension.] Rewriting R. H. S in the above expression, by partial fractions, $i$. e.

$$
\frac{1}{a} \frac{r^{m}}{\left(r-r_{1}\right)\left(r-r_{2}\right) \cdots\left(r-r_{n}\right)}=\sum_{i=1}^{n} \frac{A_{i}}{\left(r-r_{i}\right)}
$$

where

$$
\begin{aligned}
A_{i} & =\frac{r_{i}^{m}}{a} \prod_{j=1}^{n} \frac{1}{\left(r_{i}-r_{j}\right)} \quad j \neq i \\
& =\frac{1}{(m-1) \kappa_{i}}
\end{aligned}
$$

and $\kappa_{i}$ is the surface gravity of each of the horizons. Substituting the above expressions in equation (33), we get,

$$
B\left(r_{s}, r_{f}\right)= \pm \frac{E}{m-1} \sum_{i=1}^{n} \frac{1}{\kappa_{i}} \int_{r_{s}}^{r_{f}} \frac{d r}{r-r_{i}}
$$

The sign ambiguity is related to the outgoing $\left(\partial S_{0} / \partial r>\right.$ $0)$ or ingoing $\left(\partial S_{0} / \partial r<0\right)$ nature of the particle. Unlike space-times with single horizon $[18,19]$, space-times with multiple horizons have many possible ways of choosing initial $\left(r_{1}\right)$ and final $\left(r_{2}\right)$ points. However, we will consider a particular situation of the particle crossing all the horizons, $i$. e. $r_{s}<r_{1}$ and $r_{f}>r_{n}$. [This result can be used to obtain other possible cases.]

For an outgoing particle $\left(\partial S_{0} / \partial r>0\right)$ w.r.t the horizon $\left(r_{1}\right)$, the contribution to $S_{0}$ is

$$
\begin{aligned}
S_{0}(\text { emission }) & =-\frac{E}{(m-1) \kappa_{1}} \int_{r_{1}-\epsilon}^{r_{1}+\epsilon} \frac{d r}{r-r_{1}} \\
& -\frac{E}{(m-1) \kappa_{2}} \int_{r_{2}+\epsilon}^{r_{2}-\epsilon} \frac{d r}{r-r_{2}}-\cdots \\
& -\frac{E}{(m-1) \kappa_{n}} \int_{r_{n}+\epsilon}^{r_{n}-\epsilon} \frac{d r}{r-r_{n}}+\text { real part } \\
& =i \frac{\pi E}{(m-1) \kappa_{\mathrm{eff}}}+\text { real part }
\end{aligned}
$$


where

\section{B. Spherically Symmetric SdS coordinate}

$$
\frac{1}{\kappa_{\mathrm{eff}}}=\sum_{i=1}^{n} \frac{1}{\kappa_{i}}
$$

and the "-" sign in-front of the integrals correspond to the condition that $\partial S_{0} / \partial r>0$ at $r=r_{s}<r_{1}$ and $\partial S_{0} / \partial r<0$ at $r=r_{f}>r_{n}$ and the integrals are evaluated by taking the contours to lie in the upper complex plane. It should be noted that the above expression can also be considered as the action for the ingoing particles w.r.t the horizon $r_{n}$. [Note that we have assumed all $r_{i}$ 's to be positive, and hence $S_{0}$ will have imaginary contribution from all of them. For SdS, which we consider in detail, one of the roots of $g(r)$ is negative and will not contribute to $S_{0}$.]

Using the above procedure, the action for an ingoing particle $\left(\partial S_{0} / \partial r<0\right)$ w.r.t the horizon $r_{1}$ is given by

$$
\begin{aligned}
S_{0}(\text { absorption }) & =-\frac{E}{(m-1) \kappa_{1}} \int_{r_{1}+\epsilon}^{r_{1}-\epsilon} \frac{d r}{r-r_{1}} \\
& -\frac{E}{(m-1) \kappa_{2}} \int_{r_{2}-\epsilon}^{r_{2}+\epsilon} \frac{d r}{r-r_{2}}-\cdots \\
& -\frac{E}{(m-1) \kappa_{n}} \int_{r_{n}-\epsilon}^{r_{n}+\epsilon} \frac{d r}{r-r_{n}}+\text { real part } \\
& =-i \frac{\pi E}{(m-1) \kappa_{\mathrm{eff}}}+\text { real part }
\end{aligned}
$$

the last expression is obtained by evaluating the integrals with the contours taken in the lower half-plane. Taking the modulus square of equations $(37,39)$, we get

$$
P[\text { emission }]=\exp \left[-\frac{4 \pi E}{(m-1) \kappa_{\mathrm{eff}}}\right] P[\text { absorption }] .
$$

The exponential dependence on the energy allows one to give a thermal interpretation for the above result. The temperature of the emission spectrum is given by

$$
\beta^{-1}=\frac{(m-1)}{4 \pi} \kappa_{\mathrm{eff}} .
$$

The above result shows that the spectrum of particles created in a spherically symmetric space-time with multiple horizons is thermal and the temperature is proportional to the inverse harmonic sum of surface gravity of each horizon. The above result can be interpreted as a particle propagating from inside the horizon $\left(r_{1}\right)$ to outside the horizon $\left(r_{n}\right)$ picks up $\exp \left(-\beta_{i} E\right)$ at each horizon, resulting in $\exp [-\beta E)$. [Note that, we have neglected the back-scattering of the particles from the horizon.] The above analysis can be performed to Painlevé and Lemaitre coordinates for these multiple-horizons spacetimes as discussed in Appendix (A). In the next section, we explicitly demonstrate the above result for three different coordinate systems of SdS space-time.
For a quantum field propagating in spherically symmetric coordinate system of SdS space-time, the field equation is given by (29) where $g(r)$ is given by (16). In the previous section we have assumed that all the roots of $g(r)$ are positive and hence $S_{0}$ has contributions from all $r_{i}$ 's. However, for SdS, one of the roots of $g(r)$ is negative and the results in the previous section does not follow automatically.

Making the usual semi-classical ansatz for $\Psi$, and expanding $S$ in the powers of $\hbar$, we get

$$
\begin{aligned}
B(r) & = \pm \int^{r} \frac{d r}{g(r)} \sqrt{E^{2}-L^{2} / r^{2} g(r)} \\
& = \pm E \int^{r} \frac{d r}{1-2 M / r-r^{2} / l^{2}}
\end{aligned}
$$

where the last expression is obtained by setting $L=0$. After a bit of lengthy algebra, it can be shown that

$$
\begin{aligned}
\left(1-\frac{2 M}{r}-\frac{r^{2}}{l^{2}}\right)^{-1} & =\frac{\alpha}{2 \kappa_{h}\left(r-r_{h}\right)}-\frac{\alpha}{2 \kappa_{c}\left(r-r_{c}\right)} \\
& -\frac{\alpha}{2}\left(\frac{1}{\kappa_{h}}-\frac{1}{\kappa_{c}}\right) \frac{1}{r+r_{c}+r_{h}}
\end{aligned}
$$

where $\kappa_{h}, \kappa_{c}$ are given by expressions $(20,21)$ and $r_{h}, r_{c}$ are given by $(17,18)$. Substituting the above expression in equation (42), we get,

$$
\begin{aligned}
B(r) & = \pm \frac{E \alpha}{2 \kappa_{h}} \int^{r} \frac{d r}{r-r_{h}} \mp \frac{E \alpha}{2 \kappa_{c}} \int^{r} \frac{d r}{r-r_{c}} \\
& \mp \frac{E \alpha}{2}\left(\frac{1}{\kappa_{h}}-\frac{1}{\kappa_{c}}\right) \int^{r} \frac{d r}{r+r_{c}+r_{h}}
\end{aligned}
$$

The sign ambiguity is related to the outgoing $\left(\partial S_{0} / \partial r>\right.$ $0)$ or ingoing $\left(\partial S_{0} / \partial r<0\right)$ nature of the particle. Unlike space-times with single horizon [18, 19], SdS has many possible ways of choosing the initial $\left(r_{1}\right)$ and the final $\left(r_{2}\right)$ points. Following scenarios will be of our interest:

1. $r_{1}<r_{h}$ and $r_{2}>r_{c}$ : The first two integrals in the R. H. S of equation (44) does not exist.

2. $r_{1}<r_{h}$ and $r_{2}<r_{c}$ : The first integral in the R. $\mathrm{H}$. S of above expression does not exist since the denominator vanishes at $r=r_{h}$.

3. $r_{1}>r_{h}$ and $r_{2}>r_{c}$ : The second integral in the $\mathrm{R}$. $\mathrm{H}$. S of above expression does not exist since the denominator vanishes at $r=r_{h}$.

In what follows, we shall obtain the spectrum of particles produced in the first scenario. [The other two cases follows automatically from this discussion.]

In the first case, both the points are outside the region $\left(r_{h}, r_{c}\right)$. Hence, the contribution to the probability amplitude for emission and absorption will be due to both the horizons - event and cosmological. For an outgoing 
particle $\left(\partial S_{0} / \partial r>0\right)$ w.r.t the event horizon $\left(r_{h}\right)$, the contribution to $S_{0}$ is

$$
\begin{aligned}
S_{0}(\text { emission }) & =-\frac{E \alpha}{2 \kappa_{h}} \int_{r_{h}-\epsilon}^{r_{h}+\epsilon} \frac{d r}{r-r_{h}} \\
& -\frac{E \alpha}{2 \kappa_{c}} \int_{r_{c}+\epsilon}^{r_{c}-\epsilon} \frac{d r}{r-r_{c}}+\text { real part } \\
& =i \frac{\pi E \alpha}{2 \kappa_{\mathrm{eff}}}+\text { real part }
\end{aligned}
$$

where

$$
\kappa_{\mathrm{eff}}=\left[\frac{1}{\kappa_{h}}+\frac{1}{\kappa_{c}}\right]^{-1},
$$

and the "-" sign in-front of the integrals correspond to the condition that $\partial S_{0} / \partial r>0$ at $r=r_{1}<r_{h}$ and $\partial S_{0} / \partial r<0$ at $r=r_{2}>r_{c}$ and the integrals are evaluated by taking the contours to lie in the upper complex plane. It should be noted that the above expression can also be considered as the action for the ingoing particles w.r.t the cosmological horizon $\left(r_{h}\right)$.

Using the above procedure, the action for an ingoing particle $\left(\partial S_{0} / \partial r<0\right)$ w.r.t the event horizon $\left(r_{c}\right)$ is

$$
\begin{aligned}
S_{0}(\text { absorption }) & =-\frac{E \alpha}{2 \kappa_{h}} \int_{r_{h}+\epsilon}^{r_{h}-\epsilon} \frac{d r}{r-r_{h}} \\
& -\frac{E \alpha}{2 \kappa_{c}} \int_{r_{c}-\epsilon}^{r_{c}+\epsilon} \frac{d r}{r-r_{c}}+\text { real part }(48) \\
& =-i \frac{\pi E \alpha}{2 \kappa_{\mathrm{eff}}}+\text { real part }
\end{aligned}
$$

where, the integrals are evaluated by taking the contour in the lower half-plane. Taking the modulus square, we get

$$
P[\text { emission }]=\exp \left[-\frac{2 \pi E \alpha}{\kappa_{\text {eff }}}\right] P[\text { absorption }] .
$$

The exponential dependence on the energy allows one to give a thermal interpretation for the above result. The temperature of the emission spectrum is given by

$$
\beta^{-1}=\frac{1}{2 \pi \alpha} \frac{\kappa_{h} \kappa_{c}}{\kappa_{h}+\kappa_{c}} .
$$

Following features are note-worthy regarding this result:

(i) The equilibrium temperature in $\mathrm{SdS}$ is inversely proportional to the harmonic mean of the two horizons. [The range of $\kappa_{\text {eff }}$ is $(1 / l)<\kappa_{\text {eff }}<(\sqrt{3} l)$.]

(ii) In this case, radiation is propagating inwards from the cosmological horizon and outwards from the blackhole horizon. [We have neglected the scattering of the radiation from these horizons.] Thus, there is a constant flux of radiation flowing between these horizons and a static observer is in a thermal bath of radiation with the above temperature. [This result has been obtained in Ref. [15] from Euclidean quantum gravity point-of-view.] (ii) In the limit of $1 / l \rightarrow 0$, it reproduces Hawking temperature for a Schwarzschild black-hole in asymptotically flat space-time.

(iii) In the limit of $M \rightarrow 0$, this reproduces GibbonsHawking temperature for a vacuum de Sitter space-time.

(iv) In the Nariai limit $(y \rightarrow 1 / 27)$, the event and cosmological horizons coincide. Thus, the results obtained for space-times with multiple-horizons can not be applied. In the Nariai limit, using the analysis performed in Ref. [18] and Appendix A, we get [24]

$$
\beta_{\text {Nariai }}^{-1}=\frac{\sqrt{3}}{2 \pi l} .
$$

\section{Painlevé and Lemaitre SdS coordinate}

Let us now consider scalar field propagating in Painlevé coordinate system of SdS space-time. Retracing the steps as discussed in appendix (A), we get (for $l=0$ ),

$$
B(r)=E \int^{r} d r \frac{\sqrt{2 M / r+r^{2} / l^{2}} \pm 1}{1-2 M / r-r^{2} / l^{2}}
$$

As discussed in section (II B), Painlevé coordinate covers regions II and III. In the Painlevé coordinate system, as opposed to spherically symmetric coordinate, there are only two possible ways of choosing the initial $\left(r_{1}\right)$ and final $\left(r_{2}\right)$ points: (i) both the points are on one side of $r_{h}$ and (ii) the two points lie on the opposite sides of the event horizon.

For an outgoing particle $\left(\partial S_{0} / \partial r>0\right)$ w.r.t the event horizon $\left(r_{c}\right)$, the contribution to $S_{0}$ is

$$
\begin{aligned}
S_{0}[\text { emission }] & =-\frac{E \alpha}{\kappa_{h}} \int_{r_{h}-\epsilon}^{r_{h}+\epsilon} \frac{d r}{r-r_{h}}+\text { real terms } \\
& =i \frac{\pi E \alpha}{\kappa_{h}}+\text { real terms }
\end{aligned}
$$

In order to obtain the action for absorption of particles corresponding to the ingoing particles $\left[\left(\partial S_{0} / \partial x\right)<0\right]$, we have to repeat the above calculation for the lower sign of the metric given in equation (25). In this case, it is easy to see that the only singular solution corresponds to in-going particles and we get

$$
S_{0}[\text { absorption }]=-\frac{\pi E \alpha}{\kappa_{h}}+\text { real terms }
$$

Constructing the semi-classical propagator in the usual manner and taking the modulus square we obtain the probability. From the earlier discussion, we know that in calculating the probability of absorption/emission there is an extra contribution to the probability is from four sets of complex paths satisfying the semi-classical ansatz. Taking this into account, we get

$$
\beta^{-1}=\frac{\kappa_{h}}{2 \pi \alpha}
$$


In the case of Lemaitre coordinates, repeating the above procedure and it is easy to show that the temperature of the radiation is same as that obtained in the case of Painlevé coordinates and case II of Spherically symmetric coordinates. The Painlevé and Lemaitre observers will be in thermal equilibrium with the above temperature.

\section{BOGOLIUBOV COEFFICIENTS FOR SPACE-TIMES WITH MULTIPLE HORIZONS}

In this section, we extend the analysis of appendix (B) and obtain Bogoliubov coefficients for space-times with multiple horizons. The equation of the motion for the scalar field in the spherically symmetric coordinate is given by equation (29). Near each of the horizons, the solution to the wave equation is given by

$$
\Psi=C_{1}^{i} \exp (-i E t)\left(x_{i}\right)^{i E / R\left(r_{i}\right)},
$$

where $C_{1}^{i}$ is an arbitrary constant. $\Psi^{*}$ is also a solution to the scalar field equation (29). For each horizon, we can write the two sets of modes as

$$
\begin{aligned}
& \Psi\left(x_{i}<0\right)=C_{1}^{i} \psi\left(-x_{i}\right)+C_{2}^{i} \psi^{*}\left(-x_{i}\right), \\
& \Psi\left(x_{i}>0\right)=C_{\alpha}^{i} \psi\left(x_{i}\right)+C_{\beta}^{i} \psi^{*}\left(x_{i}\right),
\end{aligned}
$$

where $C_{1}^{i}, C_{2}^{i}, C_{\alpha}^{i}$ and $C_{\beta}^{i}$ are constants to be determined. We obtain the relation between the different constants similar to the single horizon case. They are given by

$$
\begin{aligned}
C_{\alpha}^{i} & =C_{1}^{i} \exp \left[\pi E / R\left(r_{i}\right)\right] \\
C_{\beta}^{i} & =C_{2}^{i} \exp \left[-\pi E / R\left(r_{i}\right)\right] .
\end{aligned}
$$

This leaves us with identifying relation between the two constants $\left(C_{1}^{i}, C_{2}^{i}\right)$ to the left of the horizon $r_{i}$. For space-times with single horizon, we assumed that the net current to the left of the horizon is zero. However, for space-times with multiple horizon, the above assumption is not valid.

In order to see this explicitly, let us consider quantum field propagating in spherically symmetric coordinate of SdS which has two horizons - event and cosmological. Let $r_{i}$ be the cosmological horizon. To the left of the cosmological horizon, the net-current is non-zero due to the presence of event horizon which produces thermal radiation. [Here we are assuming that the back-scattering of the particles from the event-horizons can be neglected.] In this case, $C_{1}^{i}$ and $C_{2}^{i}$ are related by

$$
\left|C_{1}^{i}\right|^{2}=\exp \left(-2 \pi \alpha E / \kappa_{h}\right)\left|C_{2}^{i}\right|^{2} .
$$

This gives,

$$
\left|\frac{C_{\beta}^{i}}{C_{\alpha}^{i}}\right|^{2}=\exp \left[-2 \pi \alpha E / \kappa_{\mathrm{eff}}\right] .
$$

Interpreting $\left|C_{\alpha}^{i}\right|^{2} \equiv\left|\alpha_{E}^{i}\right|^{2}$ and $\left|C_{\beta}^{i}\right|^{2} \equiv\left|\beta_{E}^{i}\right|^{2}$ as Bogoliubov coefficients and using the unitary condition, we have

$$
\begin{aligned}
\left|\alpha_{E}^{i}\right|^{2} & =\frac{1}{1-\exp \left[-2 \pi \alpha E / \kappa_{\mathrm{eff}}\right]} \\
\left|\beta_{E}^{i}\right|^{2} & =\frac{1}{\exp \left[2 \pi \alpha E / \kappa_{\mathrm{eff}}\right]-1} .
\end{aligned}
$$

Following features are noteworthy regarding this result:

1. $\left|\beta_{E}^{i}\right|^{2}$ is a Planck spectrum with a temperature given by equation (51).

2. The Bogoliubov coefficients at each of the horizon are same implying that the space-time is in thermal equilibrium with temperature proportional to $\kappa_{\text {eff }}$.

3. The condition in equation (61) has been crucial in obtaining the result. The condition implies that a particle propagating from inside the horizon $\left(r_{h}\right)$ to outside the horizon $\left(r_{c}\right)$ picks up $\exp \left[-\beta_{h} E\right]$ and $\exp \left[-\beta_{c} E\right]$ resulting in $\exp [-\beta E)$.

4. In the Nariai limit, the condition (61) is not valid and we need to impose the condition (B6) (which implies that the net current to the left of the horizon is zero). In this case, the Bogoliubov coefficients are given by equations (B8) where $\kappa=l / \sqrt{3}$.

\section{ENTROPY OF SDS}

In this section, we propose a new definition of entropy for space-times with multiple horizons analogous to the entropic definition for space-times with single horizon and discuss the implications in the context of SdS.

For space-times with (compact) single horizon, the entropy is given by

$$
S(\text { single horizon })=\frac{A}{4}=c \frac{\pi}{\kappa^{2}} .
$$

where $c=1,1 / 4$ for $\mathrm{dS}$ and Schwarzschild respectively. Assuming that the relation between $S$ and $\kappa$ holds true for space-times with multiple horizons, the entropy for space-times with multiple horizons can be written as

$$
S(\text { multiple horizons })=\frac{\pi}{\kappa_{\text {eff }}^{2}},
$$

where $\kappa_{\text {eff }}$ is given by equation (38). Following features are noteworthy regarding this expression:

(i) The above expression can be treated as an entropy associated with a single (effective) horizon for spacetimes with multiple horizon. For SdS, this corresponds to the entropy of the system in thermal equilibrium with the temperature (51).

(ii) In the literature, entropy of space-times with multiple horizons is defined as the sum of entropies of individual horizons. Using our definition, the entropy will 
have extra non-zero contributions. In the case of SdS, we have

$$
S_{S d S}=\frac{\pi}{\kappa_{h}^{2}}+\frac{\pi}{\kappa_{c}^{2}}+\frac{2 \pi}{\kappa_{c} \kappa_{h}} .
$$

If we set

$$
\frac{\pi}{\kappa_{h}^{2}}=S_{h} ; \frac{\pi}{\kappa_{c}^{2}}=S_{c}
$$

Then, the expression for entropy of SdS can be written as

$$
S_{S d S}=S_{h}+S_{c}+2 \sqrt{S_{c} S_{h}},
$$

where $S_{h}, S_{c}$ are the entropies of event and cosmological horizons, respectively.

(iii) The entropy of SdS obtained in equation (66) satisfies the D-bound conjecture [22].

Following the discussion in section (II B), we know that the range of $\kappa_{\text {eff }}$ is $(1 / l, \sqrt{3} / l)$. [The range of $1 / \kappa_{\text {eff }}$ is $(l / \sqrt{3}, l)$.] Substituting the range of $\kappa_{\text {eff }}$ in the expression for entropy, we get

$$
S_{S d S}<\pi l^{2} \equiv S_{d S} .
$$

(iv) The form of the entropy in equation (68) is similar to equation (5.1) of Ref. [17]. In Ref. [17], the authors have shown that the final values of entropies of the event $\left(S_{B}\right)$ and cosmological $\left(S_{C}\right)$ horizon satisfy the inequality

$$
S_{B}+S_{C}+\sqrt{S_{B} S_{C}} \leq \pi l^{2} .
$$

In our case, the inequality (69) is satisfied for all values of entropies of the event and cosmological horizons. The D-bound we have obtained in equation (69) is a stronger inequality than that of (70).

\section{CONCLUSIONS}

In this paper, we have studied the spectrum of created particles in SdS space-time for a linear, massless scalar field using the method of complex paths. We have shown that it is possible to obtain a temperature for SdS which corresponds to system in thermal equilibrium. The equilibrium temperature is the harmonic mean of the event and cosmological horizon temperature. We have also obtained the same result by calculating the Bogoliubov coefficients using the wave-modes near the horizons.

It has been assumed in the literature that the entropy of space-times with multiple horizons is the sum of entropies of individual horizons. In this paper, we have proposed a new definition of entropy for space-times with multiple horizons analogous to the entropic definition of entropy for space-times with single horizon. We have defined the entropy for these space-times to be inversely proportional to the square of the effective surface gravity. Using this definition, we have shown that the entropy of
SdS satisfies D-bound conjecture [22]. In Ref. [25], the authors have proposed a definition for entropy outside the cosmological horizon in SdS which satisfies D-bound. It is interesting to look for the connection between the two proposed entropies.

The above result brings attention to the following interesting questions:

1. For space-times with single horizon, there is a unique way of obtaining a global coordinate system. For the space-times with multiple horizons, it has not been possible to obtain a global coordinate system. Is it possible to obtain a global coordinate system whose Euclidean metric has periodicity proportional to $\kappa_{\text {eff }}$ ?

2. Can we obtain stress tensors for SdS which corresponds to the thermal state with the temperature proportional to $\kappa_{\mathrm{eff}}$ ?

3. What kind of quantum vacuum state will this correspond to?

We hope to return to study some of these problems in the near future.

\section{Acknowledgments}

The author would like to thank T. Padmanabhan and A. J. M. Medved for useful discussions. The author gratefully acknowledges support from Fundação para a Ciência e a Tecnologia (Portugal) under the grant SFRH/BI/9622/2002.

\section{APPENDIX A}

In this appendix, we generalize the results of Ref. [19] to two non-singular coordinate systems - Painlevé and Lemaitre - of a a general spherically symmetric spacetime (with single horizon). We show that the temperature associated with the radiation in the these two coordinate systems, for space-times with single horizon, are same as the spherically symmetric coordinate system. These results can be extended to space-times with multiple horizons where $g(r)$ is given by equation (7).

In the context of method of complex paths, these two coordinate systems possess interesting feature of double mapping of the paths. In the following, we briefly describe the multiple of mapping of space-time and measure of these paths. In Refs. [19], it was argued that, the family of complex paths, used to calculate the emission/absorption probability, takes into account of all paths irrespective of the multiple mapping of that part/whole of the space-time.

Let us consider coordinate system like Painlevé where whole of the space-time is doubly mapped w.r.t the spherically symmetric coordinate. The space-time has two distinct $R$ and $T$ regions. Hence, the complex paths will 
have equal contributions from the both of these, which do not any point in common. Hence, the contribution to the amplitude of emission/absorption by these two paths will be mutually exclusive.

In the case of coordinate systems where the part of the space-time is doubly mapped $w . r . t$. the spherically symmetric coordinate (as in the case of Lemaitre coordinate), it is always possible to find one point that is common to the paths contributing to absorption/emission. Hence, these paths are not mutually exclusive. These paths, on the other hand, will be mutually exclusive when one considers the probability amplitude - which is the important quantity in our approach. Hence, the action we obtain by regularizing the singularity and the resulting probability amplitude, for absorption/emission, will have equal contributions from both these paths.

\section{Painlevé coordinate system}

Let us consider a quantum field propagating in the Painlevé line-element (10) with the "+" signature in the cross term. The equation for a scalar field propagating in general Painlevé coordinate is given by

$$
\begin{aligned}
& r^{2} \frac{\partial^{2} \Psi}{\partial t_{P}^{2}}+2 r^{2} \sqrt{1-g(r)} \frac{\partial^{2} \Psi}{\partial r \partial t_{P}}+\frac{d}{d r}\left[r^{2} g(r)\right] \frac{\partial \Psi}{\partial r}(\mathrm{~A} 1) \\
& -r^{2} g(r) \frac{\partial^{2} \Psi}{\partial r^{2}}+\frac{d}{d r}\left[r^{2} \sqrt{1-g(r)}\right] \frac{\partial \Psi}{\partial t_{P}}=-L^{2} \Psi,
\end{aligned}
$$

where $\Phi\left(x^{\mu}\right)=\Psi\left(t_{P}, r\right) Y_{l m}(\theta, \phi)$. Making the standard semi-classical ansatz for $\Psi$ and expanding $S$ in powers of $\hbar$ as discussed in section (III A), we get, to the lowest order,

$$
\begin{aligned}
&-\left(\frac{\partial S_{0}}{\partial t_{P}}\right)^{2}+2 \sqrt{1-g(r)}\left(\frac{\partial S_{0}}{\partial r}\right)\left(\frac{\partial S_{0}}{\partial t_{P}}\right) \\
&+g(r)\left(\frac{\partial S_{0}}{\partial r}\right)^{2}+\frac{L^{2}}{r^{2}}=0 .(\mathrm{A} 2)
\end{aligned}
$$

The above equation is the Hamilton-Jacobi equation of massless particle propagating in general Painlevé lineelement (10). Substituting the ansatz, $S_{0}=-E t_{P}+B(r)$ in the above expression, we get

$$
\begin{aligned}
\frac{d B}{d r} & =-\frac{E \sqrt{1-g(r)}}{g(r)} \\
& \pm \frac{\sqrt{4 E^{2}[1-g(r)]-4 g(r)\left[E^{2}-L^{2} / r^{2}\right]}}{g(r)}
\end{aligned}
$$

It is easy to see that near the horizon, the presence of the $L^{2}$ term can be neglected since it is multiplied by $g(r)$. Thus for $L=0$, we have,

$$
\begin{aligned}
B(r) & =E \int^{r} d r \frac{\sqrt{1-g(r)} \pm 1}{g(r)} \\
& =E \int^{r} d r \frac{\sqrt{1-g(r)}}{g(r)} \pm E \int^{r} \frac{d r}{g(r)} .
\end{aligned}
$$

For space-times with single horizon, one can expand $g(r)$ around the horizon as $g(r)=R\left(r_{0}\right)\left(r-r_{0}\right)$. The numerator in the first integral (in the R. H. S) of the above expression can be approximated to unity near the horizon. Noticing that the denominator is singular at $r=r_{0}$ only for the positive sign, we get the action for the outgoing particle as

$$
\begin{aligned}
\left.S_{0} \text { [emission }\right] & =-2 E \int_{r_{0}-\epsilon}^{r_{0}+\epsilon} \frac{d r}{g(r)}+\text { real part } \\
& =+\frac{2 i \pi E}{R\left(r_{0}\right)}+\text { real part } .
\end{aligned}
$$

In order to obtain the action for absorption of particles corresponding to the ingoing particles $\left[\left(\partial S_{0} / \partial r\right)<0\right]$, we have to repeat the above calculation for the metric (10) with "-" sign in the cross term. In this case, it is easy to see that the only singular solution corresponds to ingoing particles and so

$$
S_{0}[\text { absorption }]=-\frac{2 i \pi E}{R\left(r_{0}\right)}+\text { real part }
$$

Constructing the semi-classical propagator in the usual manner and taking the modulus square we obtain the probability. Extending the double mapping of the paths to the generalized Painlevé coordinates and squaring the modulus to get the probability, we get the temperature associated with the quantum fields propagating in generalized Painlevé coordinates to be

$$
\beta^{-1}=\frac{R\left(r_{0}\right)}{4 \pi}=\frac{\kappa\left(r_{0}\right)}{2 \pi} .
$$

For dS, $g(r)=\left(1-r^{2} / l^{2}\right), \kappa=(1 / l)$ and hence, the temperature associated with the radiation is $1 /(2 \pi l)$.

\section{Lemaitre coordinate system}

Consider a quantum field propagating in the general spherically symmetric space-times described by the Lemaitre line-element (12). The scalar field equation [for the "-" sign in the expression (11)] is given by

$$
\begin{aligned}
-\frac{\partial}{\partial t_{P}}\left[r^{2} \sqrt{1-g(r)} \frac{\partial \Psi}{\partial t_{P}}\right]+\frac{\partial}{\partial R}[ & \left.\frac{r^{2}}{\sqrt{1-g(r)}} \frac{\partial \Psi}{\partial R}\right] \quad \text { (A8) } \\
& -\sqrt{1-g(r)} L^{2} \Psi=0,
\end{aligned}
$$

where $\Phi\left(x^{\mu}\right)=\Psi\left(t_{P}, R\right) Y_{l m}(\theta, \phi)$. Noting from equation (11) that $r$ is related to $R-t_{P} \equiv U$, the above equation rewritten in terms of the light-cone coordinates $(U, V)$ translates into

$$
\begin{aligned}
& \frac{\partial}{\partial V}\left[(F(U)-1) G(U) \frac{\partial \Psi}{\partial V}\right]+\frac{\partial}{\partial U}\left[(F(U)-1) G(U) \frac{\partial \Psi}{\partial U}\right] \\
+ & \frac{\partial}{\partial V}\left[(F(U)+1) G(U) \frac{\partial \Psi}{\partial V}\right]+\frac{\partial}{\partial U}\left[(F(U)+1) G(U) \frac{\partial \Psi}{\partial V}\right] \\
- & 2 \frac{\sqrt{F(U)}}{G(U)} L^{2} \Psi=0 .
\end{aligned}
$$


Making the standard semi-classical ansatz for $\Psi$ and expanding $S$ in powers of $\hbar$, we get, to the lowest order of $S_{0}$,

$$
\begin{aligned}
& \left(\frac{\partial S_{0}}{\partial U}\right)^{2}+\left(\frac{\partial S_{0}}{\partial V}\right)^{2}+2\left(\frac{1+F(U)}{1-F(U)}\right) \frac{\partial S_{0}}{\partial U} \frac{\partial S_{0}}{\partial V} \\
& -\left(\frac{L^{2}}{1-F(U)}\right)=0 \text {. }
\end{aligned}
$$

The above equation is the Hamilton-Jacobi equation of massive particle propagating in the Lemaitre line-element (12). Substituting the ansatz, $S_{0}=-E V+B(U)$ in the above expression, we get (for $L=0$ )

$$
B(U)=E \int^{U} d U \frac{1+F(U) \pm 2 \sqrt{F(U)}}{1-F(U)} .
$$

[Near the horizon the contribution due to $L$ can be neglected since it is multiplied by $g(r)$.] Notice that the denominator is singular at $U=1$ only for the positive sign. Our interest in this exercise is to obtain the principal part of the action near the horizon $r=r_{0}$. The transformation relation (11) near the horizon translates into

$$
U=-\frac{\sqrt{1-R\left(r_{0}\right)\left(r-r_{0}\right)}}{R\left(r_{0}\right)},
$$

thus giving,

$$
F(U) \equiv 1-R\left(r_{0}\right)\left(r-r_{0}\right)=\left(\frac{R\left(r_{0}\right) U}{2}\right)^{2} .
$$

Substituting the above expression in (A11), we get

$$
\begin{aligned}
S_{0}[\text { emission }] & =-\frac{4 E}{R\left(r_{0}\right)} \int_{1-\epsilon}^{1+\epsilon} d U \frac{U}{1-U}+\text { real part } \\
& =\frac{4 i \pi E}{R\left(r_{0}\right)}+\text { real part }, \quad(\mathrm{A} 14)
\end{aligned}
$$

where $U$ is rescaled as $\left[R\left(r_{0}\right) U / 2\right] \rightarrow U$.

In order to obtain the action for absorption of particles corresponding to the ingoing particles $\left[\left(\partial S_{0} / \partial r\right)<0\right]$, we have to repeat the above calculation for the metric (12) with "+" sign of expression (11). In this case, it is easy to see that the only singular solution corresponds to ingoing particles and so

$$
S_{0}[\text { absorption }]=-\frac{4 i \pi E}{R\left(r_{0}\right)}+\text { real part . }
$$

Constructing the semi-classical propagator in the usual manner and taking the modulus square we obtain the probability. Now, extending the double mapping of the paths to the generalized Lemaitre coordinates and squaring the modulus, we get the temperature associated with the quantum scalar fields propagating in the generalized Lemaitre coordinates is given by

$$
\beta^{-1}=\frac{R\left(r_{0}\right)}{4 \pi}=\frac{\kappa\left(r_{0}\right)}{2 \pi},
$$

which is same as the expression obtained in generalized Painlevé coordinate. For dS, we get $\beta^{-1}=1 /(2 \pi l)$.

\section{APPENDIX B}

In this appendix, we recover Bogoliubov coefficients (for space-times with single horizon) solely by the mode functions of the scalar field near the horizon [27]. Let us consider a quantum field propagating in a spherically symmetric coordinate (5). The equation of motion of the scalar field $\Phi=\Psi(t, r) Y_{l m}(\theta, \phi)$ is

$$
\frac{r^{2}}{g(r)} \frac{\partial^{2} \Psi}{\partial t^{2}}-\frac{\partial}{\partial r}\left(r^{2} g(r) \frac{\partial \Psi}{\partial r}\right)+L^{2} \Psi=0 .
$$

Since, our interest is near the horizon, we can transform the above expression in terms of a new variable $x \equiv r-r_{0}$. The solution to the scalar field, close to the horizon $x=0$, is given by

$$
\Psi=C_{1} \exp (-i E t) x^{i E / R(0)},
$$

where $C_{1}$ is an arbitrary constant. Note that $\Psi^{*}$ is also a solution to the scalar field equation.

In the usual field theory description, Bogoliubov transformations relate two distinct orthonormal sets of modes which are the solutions to the equation of motion. In the case of method of complex paths, the two sets of modes correspond to $\Psi(x<0)$ and $\Psi(x>0)$. We can write the two sets of modes as

$$
\begin{aligned}
& \Psi(x<0)=C_{1} \psi(-x)+C_{2} \psi^{*}(-x), \\
& \Psi(x>0)=C_{\alpha} \psi(x)+C_{\beta} \psi^{*}(x),
\end{aligned}
$$

where $C_{1}, C_{2}, C_{\alpha}$ and $C_{\beta}$ are constants to be determined. In the region $x<0, \psi(-x)$ represents an outgoing particle from the left of the horizon. Implementing the method of complex paths by rotating in the upper complex plane, we get

$$
C_{\alpha}=C_{1} \exp [\pi E / R(0)]
$$

In the region $x<0, \psi^{*}(-x)$ represents an ingoing particle to the left of the horizon. Here again, implementing the method of complex paths by rotating in the lower complex plane, we obtain

$$
C_{\beta}=C_{2} \exp [-\pi E / R(0)] .
$$

This leaves us with identifying a relation between $C_{1}$ and $C_{2}$. In order to do this, let us consider the physical situation of particle production near the black-hole horizon. In this case, a virtual particle-antiparticle pair to the left of the horizon gets converted to a real particleantiparticle pair to the right of the horizon by the tidal action of the gravitational field implying that the total current to the left of the horizon is zero. Thus, we get

$$
\left|C_{1}\right|^{2}=\left|C_{2}\right|^{2}
$$

This implies,

$$
\left|C_{\beta}\right|^{2}=\left|C_{\alpha}\right|^{2} \exp [-2 \pi E / \kappa] .
$$


Interpreting $\left|C_{\alpha}\right|^{2} \equiv\left|\alpha_{E}\right|^{2}$ and $\left|C_{\beta}\right|^{2} \equiv\left|\beta_{E}\right|^{2}$ as the Bogoliubov coefficients and using the unitarity condition, we get

$$
\left|\alpha_{E}\right|^{2}=\frac{1}{1-\exp [-2 \pi E / \kappa]}
$$

$$
\left|\beta_{E}\right|^{2}=\frac{1}{\exp [2 \pi E / \kappa]-1} .
$$

These are the well known relations for the Bogoliubov coefficients.
[1] S. Perlmutter et al, Astro. Phys. J. 483, 565 (1997); B. Schmidt et al, Astro. Phys. J. 507, 46 (1998); A. J. Riess et al, Astron. J. 116, 1009 (1998).

[2] J. Maldacena, Adv. Theo. Math. Phys. 2, 231 (1998) [hep-th/9711200]; E. Witten, Adv. Theor. Math. Phys. 2253 (1998); S. Gubser, I. Klebanov and A. Polyakov, Phys. Lett. B 428, 105 (1998; O. Aharony, S. Gubser, J. Maldacena, H. Ooguri and Y. Oz, Phys. Rep. 323, 183 (2000) [hep-th/9905111].

[3] E. Witten, Quantum gravity in de Sitter space, hep-th/0106109.

[4] A. Strominger, The dS/CFT Correspondence,a hep-th/0106113

[5] C. M. Hull, JHEP 9807, 021 (1998) [hep-th/9806146]; Mu-In Park, Phys. Lett. B 440, 275 (1998); Nucl. Phys. B 544, 377 (1999); I. Antoniadis, P. Mazur and E. Mottola, astro-ph/9705200; A. Volovich, hep-th/0101176; V. Balasubramanian, P. Horava and D. Minic, hep-th/0103171.

[6] A. J. M. Medved, Phys. Rev. D 66, 124009 (2002).

[7] M. Parikh, Phys. Lett. B 546, 189 (2002).

[8] R. Busso, A. Maloney and A. Strominger, Phys. Rev. D 65, 104039 (2002)

[9] Kyung-Seok Cha, Bum-Hoon Lee, Chanyong Park, dS/CFT correspondence from the brick wall method, hep-th/0207194

[10] S. Nojiri and S. Odintsov, JHEP 0112, 033 (2001); Phys. Lett. B 523, 165 (2001).

[11] M. Parikh and F. Wilczek, Phys. Rev. Lett. 85, 5042 (2000).

[12] M. Spradlin, A. Strominger, A. Volovich, Les Houches Lectures on De Sitter Space, hep-th/0110007.

[13] G. W. Gibbons and S. W. Hawking, Phys. Rev. D 15, 2738 (1977); W. Hiscock, Phys. Rev. D bf 39, 1067 (1989); D. Markovic and W. Unruh, Phys. Rev. D 43, 332 (1991); Zhong Chao Wu, Gen. Rela. Gravi. 32, 1823
(2000)

[14] T. Padmanabhan, Class. Quant. Grav. 19, 5387 (2002); T. Padmanabhan, Entropy and energy of a class of spacetimes with horizon: a general derivation, gr-qc/0202080.

[15] Feng-Li Line and Chopin Soo, Class. Quant. Grav. 16, 551 (1999).

[16] S. Nojiri and S.D. Odintsov, Phys. Rev. D 59,044026 (1999); A. Bytsenko, S. Nojiri and S. Odintsov, Phys. Lett. B 444, 121 (1998); S. Nojiri ad S.D. Odintsov, Int. Journ. Mod. Phys. A 14, 1293 (1999); Int. Journ. Mod. Phys. A 15, 989 (2000).

[17] K. Maeda, T. Koike, M. Narita and A. Ishibashi, Phys. Rev. D 57, 3503 (1998).

[18] K. Srinivasan and T. Padmanabhan, Phys. Rev. D 60, 24007 (1999).

[19] S. Shankaranarayanan, K. Srinivasan and T. Padmanabhan, Mod. Phys. Lett. A 16, 571-578 (2001); S. Shankaranarayanan, T. Padmanabhan and K. Srinivasan, Class. Quant. Grav. 19, 2671 (2002).

[20] S. Shankaranarayanan, Field theoretic methods in gravity, Ph. D Thesis, IUCAA, Pune.

[21] Elias C. Vagenas, to appear in Nuo. Cimen. B., hep-th/0111047; Damien A. Easson, hep-th/0210016.

[22] R. Bousso, JHEP 0011, 038 (2000) [hep-th/0010252]; JHEP 0104, 035 (2001) [hep-th/0012052].

[23] I. D. Novikov, Communications of the Shternberg state Astronomical Institute, 132, 3-42 (1964).

[24] R. Bousso and S. W. Hawking, Phys. Rev. D 54, 6312 (1996); R. Bousso and S. W. Hawking, Phys. Rev. D 57, 2436 (1998); J. Niemeyer and R. Bousso, Phys. Rev. D 62, 023503 (2000).

[25] R. Mann and M. Ghezelbash, JHEP 01, 005 (2002).

[26] S. Shankaranarayanan and T. Padmanabhan, paper in preparation.

[27] K. Srinivasan, private communication. 